\title{
Carnets
}

Revue électronique d'études françaises de l'APEF

Première Série - $1 \mid 2009$

La mer... dans tous ses états

\section{Métaphores liquides chez Zola ou la mer dans tous ses états}

\section{Ana Clara Santos}

\section{(2) OpenEdition}

\section{Journals}

Édition électronique

URL : http://journals.openedition.org/carnets/3085

DOI : 10.4000/carnets.3085

ISSN : 1646-7698

Éditeur

APEF

Édition imprimée

Pagination : 163-174

\section{Référence électronique}

Ana Clara Santos, « Métaphores liquides chez Zola ou la mer dans tous ses états », Carnets [En ligne], Première Série - 1 | 2009, mis en ligne le 14 juin 2018, consulté le 03 mai 2019. URL : http:// journals.openedition.org/carnets/3085 ; DOI : 10.4000/carnets.3085

\section{(c) (1) (8)}

Carnets est mis à disposition selon les termes de la licence Creative Commons - Atribution - Pas d'utilisation commerciale 4.0 International. 


\section{METAPHORES LIQUIDES CHEZ ZOLA \\ Ou la mer dans tous ses états}

ana Clara Santos

Universidade do Algarve

avsantos@ualg.pt

\section{Résumé}

Ébauché dès 1880 et conçu en 1883, La joie de vivre marque la rencontre avec un décor, un petit village de pêcheurs sur la côte normande, et une crise, la menace de la mer à l'image de l'écrasement de l'homme sous le poids de la nature. Par son aspect constamment renouvelé, c'est la mer qui fascine. Source de contemplation, océan-spectacle, elle devient, par son caractère théâtral, source de plaisir et de mort. Symbole de la lutte archétypale de l'homme avec l'inconscient collectif, traduit par l'illusion de son pouvoir sur la nature déchaînée, le paysage marin établit aussi, chez Zola, des jeux de correspondance avec l'imaginaire féminin. À l'obsession des marées, signe de dévoration et de dissolution, se superpose la description des règles du personnage féminin et du cycle de son corps dans une métaphore filée autour de l'élément liquide. Les deux sont source de transformations et renouvellements perpétuels à la régulation pendulaire qui amène d'emblée une profonde réflexion sur la vie et la mort.

\section{Abstract}

Having first taken shape in 1880 and written in 1883, La joie de vivre marks the return of the author to a particular setting, a small fishing village on the coast of Normandy, and to a crisis, the threat of the sea, the overwhelming weight of nature over man. For its constantly renewed aspect, the sea is what fascinates. A source of contemplation, océan-spectacle, the sea turns, for its theatrical character, into a source both of pleasure and of death. A symbol of the archetypal struggle of man against the collective unconscious, translated through the illusion of its power over unrestrained nature, the maritime landscape in Zola also establishes games of correspondence with the feminine imagery. The obsession of the sea tides, a sign of devouring and dissolution, is overcome by the description of the period of the feminine character and of the cycles of her body in a metaphor structured around the liquid element. Both are sources of transformations and perpetual renewal, in the pendulous regulation which leads straight to a deep reflection on life and death.

Mots-clés: Zola, Naturalisme, Mer, Vie, Mort

Keywords: Zola, Naturalism, Sea, Life, Death 
Comme dans tous les romans de la saga des Rougon-Macquart, Zola projette pour celui-ci - La joie de vivre - son protocole d'écriture défini au préalable basé sur la dite "méthode naturaliste" qui comporte le recueil méthodique de documents avec des visites sur les lieux, des enquêtes sur le terrain, le recours à des spécialistes et à des livres de spécialité, l'établissement d'une Ébauche susceptible de fixer les contours généraux de l'oeuvre, la rédaction de plans plus précis, ainsi qu'un chronogramme des personnages. Si on rajoute, à cette "méthode", la projection de la rédaction définitive, grosso modo, en l'espace d'un an, fruit de la production romanesque de trois ou quatre pages par jour, on aura ainsi fait le tour des règles imposées par l'auteur lors de la création de tous les romans des Rougon-Macquart. Or, justement, il est intéressant de remarquer que celui de La joie de vivre fait exception à la règle. Pour la première fois, confronté aux imprévisibilités de la vie réelle, l'auteur ne suit plus son protocole d'écriture. Des évènements tragiques tels que la mort de Duranty, le 9 avril 1880, de Flaubert, le 8 mai, et, surtout, celle de sa mère, le 17 octobre de la même année, ont sûrement déclenché chez l'auteur de graves angoisses et la hantise de la mort dont parle Goncourt dans son Journal:

Ce soir, après dîner, au pied de ce lit qui a l'air d'un lit d'archevêque dans un drame de Boulevard, Zola se met à parler, selon son habitude, de la mort. Cette idée de la mort est plus fréquente encore chez lui depuis la mort de sa mère; et après un silence, il ajoute que cette mort a fait un trou dans le nihilisme de ses convictions religieuses, tant il lui est affreux de penser à une séparation éternelle. Et il dit que ce hantement de la mort et, peut-être, une évolution des idées philosophiques amenée par le décès d'un être cher, il songe à l'introduire dans un roman, auquel il donnerait un titre comme La douleur (Goncourt, 1956: 236).

On le sait, Zola aurait projeté depuis la publication de Nana au mois de mars 1880 à écrire "un roman intime, à peu de personnages" qui prendrait, en fait, une nouvelle orientation, "une sorte de réaction contre [ses] œuvres antérieures"1 où la réflexion sur I'homme face au sacrifice et à la mort serait susceptible d'illustrer ses partis pris pour une nouvelle conception de la description annoncée dans Les romanciers naturalistes:

\footnotetext{
${ }^{1}$ Ces propos tenus par Zola à son ami journaliste Fernand Xau sont repris par Colette Becker et Philippe Hamon dans la préface de l'édition du Livre de Poche, de 2005, de La joie de vivre (édition utilisée par nous-mêmes dans cette petite étude). Ces spécialistes de l'œuvre zolienne expliquent ainsi la genèse du roman zolien: "Pourtant, tout avait bien commencé: Nana est sorti en mars 1880. Aucun des anciens plans généraux des RougonMacquart, rédigés par Zola en 1868-1869, ne prévoyait de faire de Pauline l'héroïne d'un roman important: elle n'apparaissait que très épisodiquement dans Le Ventre de Paris, grosse fille placide jouant à la poupée ou avec son chat. C'est vers mars-avril 1880 que Zola songe, en antithèse du roman de Nana, à faire un roman moins 'documentaire', plus 'philosophique' et 'psychologique', moins ancré dans la réalité du Second Empire, un roman qui traiterait de l'homme face à la douleur et à la mort plus que de l'homme dans ses appétits, dans sa lutte pour le pouvoir et la conquête des biens matériels" (Zola, 2005: 8).
} 
Les moindres détails s'animent comme d'un tremblement intérieur. Les pages deviennent de véritables créatures, toutes pantelantes de leur outrance à vivre [...] On dirait la nature racontée par deux voyants, animée, exaltée, les cailloux ayant des sentiments d'être vivants, les personnages donnant de leur tristesse ou de leur joie aux horizons. L'œuvre entière devenait une sorte de vaste névrose (Zola, 1895: 226).

Cette rencontre avec l'exaltation de la nature, à la manière des Goncourt, en parfaite harmonie avec l'être vivant, marquée par une prédilection envers les teintes impressionnistes, nuancées par de petites touches, ici et là, dans un jeu de contrastes qui pose la tonalité tantôt sur la lumière et les couleurs tendres, tantôt sur l'opacité des couleurs sombres et violentes, explique sans doute le changement d'orientation entre les deux ébauches élaborées pour le roman en l'espace de trois ans. Alors que la première Ébauche (1880) avait pour but essentiel la question du sacrifice associé à une héroïne, Pauline Hédouin, capable de cacher l'adultère et le crime, en opposition avec l'héroïne du roman précédent, Nana, la deuxième Ébauche (1883), créée après Pot-Bouille et Au Bonheur des Dames, focalise son attention sur le personnage de Lazare et le contraste entre celui-ci et Pauline, entre une vision angoissante de la mort et la joie de vivre, titre choisi pour le roman. Mais plus important encore, alors que la première Ébauche situe l'action dans une petite ville de province, loin de la mer, ravagée par la fièvre typhoïde où la principale entreprise est celle de la construction d'une grande maison édifiée au fur et à mesure de la progression du roman, la deuxième Ébauche place l'action au bord de l'océan et privilégie la lutte de l'homme contre les éléments naturels.

La mer devient donc dans le roman, par ce processus de recréation et de changement de direction opéré par l'auteur, un élément clé autour duquel tout gravite. Cette mer qui inonde maintenant l'écriture zolienne à laquelle sont associées toutes les métaphores filées de la maternité et de l'élément liquide, comme on verra plus loin, est tout d'abord le synonyme d'une apologie de son homophone "mère". Tout semble se jouer entre une présence, celle de la mer, vivante et omniprésente dans le roman, et une absence, celle de la mère de l'écrivain qui amène, avec sa disparition, l'interruption de la rédaction du roman dans une première phase. A partir de là, la rédaction de l'œuvre se fait en l'espace de quelques mois, entre le mois d'avril et le mois de novembre de l'année 1883, entre Médan et les bords de mer en Bretagne.

En regardant de plus près le roman à la lumière de ce qui vient d'être dit, on peut affirmer que La joie de vivre est composé sur un principe cyclothymique par lequel s'accentuent les alternances entre les présences et les absences, les moments d'angoisse et les moments de joie, à l'image des montées et des descentes des marées hautes et des marées basses de l'océan. Tel un cycle des humeurs analysées au bon gré du temps qui 
passe ou de l'eau qui coule en perpétuel mouvement, l'esthétique romanesque zolienne se rapproche ici d'une position philosophique assumée par Schopenhauer à laquelle l'auteur naturaliste ne resta pas indifférent ${ }^{2}$. Ayant voulu faire de ce roman au titre profondément ironique (la joie de vivre versus l'angoisse et la douleur de mourir), comme il déclare dans l'Ébauche, l'image d'un "petit village mangé par la mer", incarnation symbolique de "l'image de l'écrasement du monde", rien d'étonnant que l'incipit du roman pose l'encadrement naturel sous une tonalité majoritairement noire et menaçante, sombre et mélancolique:

Ses craintes le reprenaient, en face du ciel livide, où le vent d'ouest emportait de grands nuages noirs, comme des haillons de suie, dont les déchirures traînaient au loin dans la mer. C'était une de ces tempêtes de mars, lorsque les marées de l'équinoxe battent furieusement les côtes. Le flot, qui commençait seulement à monter, ne mettait encore sur l'horizon qu'une barre blanche, une écume mince et perdue; et la plage, si largement découverte ce jour-là, cette lieue de rochers et d'algues sombres, cette plaine rase, salie de flaques, tachée de deuil, prenait une mélancolie affreuse, sous le crépuscule tombant de la fuite épouvantée des nuages (Zola, 2005: 30).

La description du village imaginaire de Bonneville ${ }^{3}$ se fait d'emblée en édifiant le décor par des jeux de contrastes, de réduction du terrain d'action, circonscrit par la force des éléments naturels qui limitent, en quelque sorte, l'espace de représentation du drame mis sous les yeux du lecteur:

Cette route dévalait entre deux falaises, on aurait dit un coup de hache dans le roc, une fente qui avait laissé couler les quelques mètres de terre, où se trouvaient plantées les vingt-cinq à trente masures de Bonneville. Chaque marée semblait devoir les écraser contre la rampe, sur leur lit étroit de galets. À gauche, il y avait un petit port d'échouage, une bande de sable, où des hommes hissaient à cris réguliers une dizaine de barques. Ils n'étaient pas deux cents habitants, ils vivaient de la mer, fort mal, collés à leur rocher avec un entêtement stupide de mollusques. Et, au-dessus des misérables toits, défoncés chaque hiver par les vagues, on ne voyait sur les

\footnotetext{
${ }^{2}$ Zola ne resta pas indifférent à l'œuvre et à la pensée de Schopenhauer diffusée à l'époque. Selon Philippe Hamon, dans la préface de La joie de vivre, Zola alla même jusqu'à prélever une série d'expressions et de notions dans l'édition de Jean Bourdeau, Pensées, maximes et fragments de Schopenhauer (1880) telles que "la vallée de larmes, la joie de vivre, l'espoir du néant, le vieux cynique, la sombre mort, le tourment de l'existence, la misère du monde, le repas sacré du néant, le triste monde" (Zola, 2005: 11).

${ }^{3}$ Bien qu'inventé de toutes pièces par l'auteur, Bonneville a des attaches profondes avec son environnement réel, notamment avec Saint-Aubin, Grand-Camp et Vierville comme Zola l'explique dans une lettre adressée à Van Santen Kolff le 7 juillet 1887: "Je connais la côte normande, de Lion-sur-Mer à Cherbourg, pour avoir passé des étés dans plusieurs stations, notamment à Saint-Aubin, en 75, et à Grand-Camp, en 1881. Je suis allé en voiture de village en village, et je puis même vous dire que Bonneville n'est autre que Vierville (entre Port-enBassin et Grand-Camp), un Vierville arrangé. Le plus souvent, je crée ainsi le hameau dont j'ai besoin, en gardant les villes voisines, telles qu'elles existent".
} 
falaises, à demi-pente, que l'église à droite, et que la maison des Chanteau à gauche, séparées par le ravin de la route. C'était là tout Bonneville (Zola, 2005: 30-31).

Tout cela, au service de la verbalisation minutieuse d'un plan préétabli avant l'acte d'écrire, comme à l'habitude de la méthode naturaliste zolienne. La mer est décrite par petites touches de façon à faire apparaître, au bout des premiers chapitres du roman, ses divers angles et ses multiples facettes qui, animés d'une vie et d'un mouvement perpétuel, constituent la toile de fond d'un paysage narratif qui se veut avant tout source de contemplation:

La mer, cependant, battait deux fois par jour Bonneville de l'éternel balancement de sa houle, et Pauline grandissait dans le spectacle de l'immense horizon [...] son unique recréation était de regarder la mer, toujours vivante, livide par les temps noirs de décembre, d'un vert délicat de moire changeante aux premiers soleils de mai (Zola, 2005: 87-88).

On pourrait dire que, dans cette première phase du roman, le paysage maritime vit et respire à l'écoute des personnages servant ainsi, sous la plume de l'écrivain, à fixer les caractères et les grandes névroses des personnages principaux, notamment l'opposition du couple idyllique Pauline-Lazare. Se dessinent alors les traits essentiels du personnage féminin caractérisé par la sagesse et la gaieté que la menace de la marée montante n'ébranle pas:

... d'Arromanches à Bonneville, elle avait à chaque minute allongé la tête hors du cabriolet, malgré le vent, pour voir la mer qui les suivait. Et, maintenant, la mer était encore là, elle serait toujours là, comme une chose à elle. Lentement, d'un regard, elle semblait en prendre possession [...] "Ça ne vous effraie pas?" [...] Non, pourquoi? ... Bien sûr que l'eau ne montera pas jusqu'ici. [...] mais elle éclat d'un bon rire. Dans son petit être réfléchi, c'était une bouffée de gaieté bruyante et saine, la gaieté d'une personne de raison que l'absurde met en joie (Zola, 2005: 41-42).

ainsi que son côté maternel, protecteur et solidaire envers les plus démunis:

Et, pendant une heure encore, la canonnade continua, chaque vague qui s'abattait la secouait d'un choc profond et sourd. II lui semblait que la maison, anéantie, écrasée de silence, s'en allait dans l'eau comme un navire, Elle avait maintenant une bonne chaleur moite, sa pensée vacillante se reportait, avec une pitié secourable, vers les pauvres gens que la mer, en bas, chassait de leurs couvertures (Zola, 2005: 64). 
Le même procédé est utilisé pour la fixation du caractère du personnage masculin, très tôt fragilisé par l'angoisse et la peur de la mort"

La mer, qui montait, avait une lamentation lointaine, pareille à un désespoir de foule pleurant sa misère. Sur l'immense horizon, noir maintenant, flambait la poussière volante des mondes. Et, dans cette plainte de la terre écrasée sous le nombre sans fin des étoiles, l'enfant crut entendre près d'elle un bruit de sanglots. "Qu'as-tu donc? es-tu malade?" II ne répondit pas, il sanglotait, la face couverte de ses mains crispées violemment, comme pour ne plus voir. Quand il put parler, il bégaya: "Oh! mourir, mourir!" (81).

Passée cette première phase de contemplation de l'océan-spectacle, le paysage maritime devient actant de l'intrigue et, par là, mythique. Déclencheur des passions, poussant les protagonistes vers la catastrophe et leur devenir, la mer se transforme en un lieu de flux et de circulation de sens ${ }^{5}$.

S'ouvrant au monde en pleine période estivale ${ }^{6}$, maintenant calme et sereine à l'eau si pure et si claire sur des plages immenses, la mer reste avant tout "symbole de la dynamique de la vie" (Chevalier, Gheerbrant, 1982: 623), lieu d'un éternel recommencement:

Et ce fut de là, en poussant une petite porte, qu'ils se trouvèrent sur les falaises, sous le ciel libre, en face de la pleine mer. Pauline avait gardé la curiosité passionnée de cette eau immense, si pure et si douce maintenant, au clair soleil de juillet. C'était toujours la mer qu'elle regardait de chaque pièce de la maison. Mais elle ne l'avait pas encore approchée, et une vie nouvelle commença, quand elle se trouva lâchée avec Lazare dans la solitude vivante des plages (Zola, 2005: 77).

Source de vie, elle est aussi source de bonheur chez ceux qui savent partir à la recherche de ses richesses et de ses mystères, mais aussi de ses potentialités nourrissantes:

\footnotetext{
${ }^{4}$ II l'avait annoncé dans l'Ébauche: “Je tiendrai beaucoup à garder mon type de l'homme du monde moderne, et hanté par la mort; et ravagé par cette obsession secrète [...]. L'influence même de la vie opère, la mort lente et quotidienne, le lent engourdissement qui arrive, et les pertes qu'on fait, la mort se dressant avec l'âge".

${ }^{5}$ Cette première acception est tout de suite ressentie par ses lecteurs et amis. Tel est le cas de Louis Desprez qui écrit justement cela à Zola dans une lettre datée du 19 février 1884: "Elle joue, cette mer, le rôle de Paris dans la Page d'amour - moins artificiellement, je l'avoue. - Le décor change comme les personnages et semble suivre la gamme de leurs passions et leurs sentiments. Mais vous voyez l'océan en terrien, avec une pointe d'hostilité comme Lazare [...]. Eh bien, on sent que l'Océan est pour vous un spectacle et pour Loti une sorte de propriété animée. Le marin a pour la mer la passion aveugle des habitants de Bonneville. II est tout fier d'elle, lorsqu'elle lui démolit son navire; vous êtes, au contraire, vous, pour les épis et les digues".

${ }^{6}$ Comme le font remarquer Philippe Hamon et Colette Becker, "le grand développement de la mode et de la vogue des bains de mer [...] date essentiellement du Second Empire en France, et se localise d'abord sur les côtes de la Manche, les plus proches de Paris (Zola, 2005: 78).
} 
Ce qui était amusant, c'était, à mer basse, de s'en aller très loin, sous les falaises: on marchait sur des sables fins, où fuyaient des crabes, on sautait de roche en roche, parmi les algues, pour éviter les ruisseaux d'eau limpide, pleins d'un frétillement d crevettes; sans parler de la pêche, des moules mangées sans pain, toutes crues, des bêtes étranges, emportées dans le coin d'un mouchoir, des trouvailles brusques, une limande égarée, un petit homard entendu au fond d'un trou (Zola, 2005: 78).

Ce premier moment annonce déjà un autre qui coïncide avec le nœud de l'action l'extraction des richesses de la mer en bénéfice de l'industrie scientifique - et fait apparaître tout le tissu symbolique de la mer et de ses homophones. En effet, tout le texte est habité et rythmé par les présences et absences des leitmotiv homophones de "mer", notamment par "mère". Disséminée dans les expériences scientifiques de Lazare lors de l'exploitation d'algues qui le poussent à distiller les "eaux mères" pour obtenir du bromure en vue de la création de l'usine du Trésor, la fonction maternelle rejaillit surtout de la figure de Pauline tour à tour "mère des bêtes"7 , des petits pauvres, du petit Paul qu'elle ressuscite ou, tout simplement, la "mère de son petit monde" (Zola, 2005: 345).

A part ses acceptions homophones, le texte engendre aussi plusieurs images sur la fonction maternelle, bonne ou mauvaise. Le mot lui-même clôt le chapitre IV ${ }^{8}$ et se profile par des images contraires, celle de la Bonne Mère et celle de la Mère Terrible où s'opposent les figures humaines - Pauline, la Bonne Mère - et animales - Minouche, la Mère Terrible. Commençons par cette dernière. Minouche, la chatte, préfigure ici la figure de la Mère Terrible qui, au détriment des pulsions sexuelles inhérentes à sa condition, nie ses fonctions maternelles les plus élémentaires, c'est-à-dire la sauvegarde de la vie de ses petits:

... Je crois qu'elle perd un peu la vue, ce qui ne l'empêche pas de se conduire comme une coquine... Imaginez-vous qu'on lui a jeté sept petits, il y a une semaine à peine. Elle en fait, elle en fait tellement, qu'on en reste consterné [...]. Eh bien, elle a encore disparu mardi, et vous la voyez qui se nettoie, elle n'est rentrée que ce matin, après trois nuits et trois jours d'abominations [...]. Du reste, il n'y avait toujours pas de plus mauvaise mère (Zola, 2005: 474).

Pauline, toujours associée au sacrifice de soi-même en bénéfice des autres, incarne parfaitement la figure de la Bonne Mère, protectrice et dévouée. Mais Zola en produit une

\footnotetext{
${ }^{7}$ Comme on peut lire à la page 101: "C'était, chez Pauline, un amour de la vie, qui débordait chaque jour davantage, qui faisait d'elle 'la mère de bêtes', comme disait sa tante. Tout ce qui vivait, tout ce qui souffrait, l'emplissait d'une tendresse active, d'une effusion de soins et de caresses. Elle avait oublié paris, il lui semblait avoir poussé là, dans ce sol rude, un souffle pur des vents de mer" (Zola, 2005: 101).

${ }^{8}$ Voici comment termine le chapitre: "La peur de la mort venait de reparaître dans son sommeil, une peur sans cause, comme sortie du néant lui-même, une peur dont le souffle glacé l'avait éveillé d'une grand frisson. Mon Dieu ! Il faudrait mourir un jour! Cela montait, l'étouffait, tandis que Pauline, qui avait reposé la tête sur l'oreiller, le regardait de son air de compassion maternelle" (Zola, 2005: 203).
} 
figure encore plus forte, caractérisée par la joie de vivre et la source de vie et du maintient de l'ordre et de l'équilibre:

Pauline restait la mère de son petit monde, soignait Chanteau qui allait mal, était obligée de suppléer Véronique dont la propreté se gâtait, sans compter Lazare et Louise qu'elle feignait de traiter en gamins turbulents pour pouvoir sourire de leurs escapades. Elle arrivait à rire plus haut qu'eux, de ce beau rire sonore qui sonnait la santé et le courage de la vie, avec des notes limpides de clairon. La maison entière s'égayait (Zola, 2005: 345-346).

Or cette image est supplantée, très vite, par une autre, celle de la liquidité qui rattache toute la symbolique féminine à ce décor accentué par l'élément fluide fondamentalement ancré sur l'obsession des marées et sa correspondance avec la description de l'énigme du corps féminin, les règles de Pauline ou l'accouchement de Louise. L'angoisse initiale lors de l'apparition de la première menstruation, d'un côté et, de l'autre, l'enthousiasme qui l'animait au cours de la formation de son corps de jeune femme, sont vite remplacés, après le "miracle de résurrection" de ce "pauvre être à peine formé [...] ce fils misérable que Louise donnait à Lazare" (Zola, 2005: 449-450), par une résignation sur son sort. Un sort qui l'empêchait d'être mère et qui vouait à l'inusité les fluides qui coulaient en elle:

Elle baissait un regard désespéré vers ses hanches, vers son ventre de vierge qui venait de tressaillir. Dans la largeur de son flanc, elle aurait tenu un fils solide et fort. C'était un regret immense de son existence manquée, de son sexe de femme qui dormirait stérile. La crise dont elle avait agonisé, pendant la nuit des noces, recommençait, en face de cette naissance. Justement, le matin, elle s'était éveillée ensanglantée du flux perdu de sa fécondité: et, à ce moment même, après les émotions de cette terrible nuit, elle le sentait couler sous elle, ainsi qu'une eau inutile. Jamais elle ne serait mère, elle aurait voulu que tout le sang de son corps s'épuisât, s'en allât de la sorte, puisqu'elle n'en pouvait faire de la vie (Zola, 2005: 450).

Mais, comme l'a si bien signalé Roger Ripoll, l'étude des "phénomènes de la menstruation obéit au désir de faire éclater les cadres de l'expérience individuelle, de montrer un être dominé par le jeu des forces naturelles vues dans toute leur ampleur et dans toute leur brutalité" (Ripoll, 1981: 695). De cette façon, les jalousies et les débordements d'amour de Pauline, les silences et les flots de paroles de Mme Chanteau, les larmes et les liquidations des diverses fortunes, marquent l'harmonie d'une symphonie jouée au rythme du renouvellement de la nature (les saisons et les marées) et celui des pulsions du désir du 
règne animal et humain où la mer et la figure féminine tiennent la cadence. Voilà la réalisation pleine, sous la plume de Zola, d'un livre de flux et de rythmes, d'“un livre de cycles au centre du cycle", comme l'affirme l'auteur de Feux et signaux de brume:

Pauline, la débâcle de la marée de sang, ne saurait vivre ailleurs qu'au bord de l'océan. De la mer qui bat indéfiniment, continûment, par périodes et catastrophes, la falaise de craie, les épis de métal, la digue de bois dur. Pauline, la marée mensuelle, est cobordante de la mer, elles débordent ensemble, elles préparent inutilement dans le cercle du temps, les vibrations et les tempêtes, la soupe prébiotique. Mer amère de larmes, mer blanche sous la pluie fine des noces, mer vineuse des nuits d'été, mer noire d'encre aux équinoxes, mer périodique et stable, le sang des métamorphoses vitales. Pauline vive née des eaux, marée de vie née de Pauline. Généreuses et génétiques [...]. Dans la circulation périodique, stable, déchirée de coupures, du liquide fondamental, prébiotique, la mer. Qui donne la pluie à la terre, et aux arbres la sève, et aux seiches leur encre, à la vigne son vin et aux femmes leur sang. Pauline mer, vierge et mère (Serres, 1975: 257).

Ce va-et-vient constant entre deux mouvements qui finissent par s'annuler et jamais atteindre leur but, permet de redimensionner la vison naturaliste zolienne qui s'attaque ici, à travers ces images construites autour de la mer et ses homophones, à ce qui est constant et reste néanmoins immuable, à ce qui est source de passion et finit impassible. La littérature tend ainsi, sous la plume zolienne, vers le tableau descriptif de la nature sous ses différentes facettes grâce à la peinture de ses mouvements alternatifs, mais toujours permanents, transférable à une réflexion philosophique sur la nature humaine dans une volonté de rendre la vie telle qu'elle est, aux emprises avec les pulsions contraires qui, tels les mouvements houleux de la mer éternelle, poussent l'homme vers la névrose, la déchéance et l'immobilité. Face à une mer toujours changeante, invitant au mouvement et à l'action, la condition humaine n'est-elle pas, pour ainsi dire, condamnée à l'impassibilité, incapable d'agir sur soi et sur son propre milieu?

Cette fatalité est bien incarnée par Chanteau, dévoré petit à petit par la goutte, pétrifié et cloué devant "cet infini bleu où passaient des voiles blanches, cette route sans bornes, ouverte devant lui qui n'était plus capable de mettre un pied devant l'autre" (Zola, 2005: 459). L'image de l'érosion constante de la mer sur la falaise de craie qui émiette, par son rythme chronique, le village de pêcheurs se voit, pour ainsi dire, transférée sur le corps même de Chanteau sur qui "la goutte chronique avait accumulé la craie à toutes les jointures, des tophus énormes s'étaient formés, perçant la peau de végétations blanchâtres" (Zola, 2005: 458). 
Beaucoup plus que dans les autres romans où quelques personnages ou groupes sociaux sont atteints de telles ankyloses de l'immobilité du corps ou de l'âme ${ }^{9}$, dans La joie de vivre ce phénomène s'intensifie et tend à se généraliser car il devient extensible à tous les personnages et même à certains animaux comme le chien Mathieu. Paralysie physique, mais aussi paralysie mentale qui empêche notamment l'homme de venir à bout des contraintes imposées par son milieu naturel en détriment de ses connaissances scientifiques. La science de Lazare restera impuissante face à la force naturelle des montées des marées de l'océan, tout comme celle de Cazeneuve sera incapable de venir à bout de "l'enflure qui monte" chez Mme Chanteau condamnée, par là, à l'inertie de son lit.

Désormais, les débordements et les flots plongent, par des mouvements symétriques, l'homme et le village dans la paralysie et l'émiettement de la chair et de la terre.

L'écrivain file ici la métaphore de la "débâcle" (c'est le titre de l'avant-dernier roman de la série), celle de la "fêlure" héréditaire qui "lézarde" l'arbre familial des RougonMacquart, celle de "l'émiettement" des choses et des êtres qui va courir dans toute sa série: sa première héroïne, qui s'appelle "Miette", apparaît dans le premier roman du cycle, La Fortune des Rougon, parmi les tombes "émiettées" du cimetière de l'aire "Saint-Mittre". Bonneville, dont le nom est aussi ironique que celui du roman, est peu à peu "mangé" et "émietté" par la mer; Lazare est caractérisé par un "émiettement de son être" (p. 307); Mme Chanteau se "dévore elle-même", en un sourd travail de jalousie et d'avarice qui "émiettait en elle les bons sentiments" (p. 240), pendant que Pauline assiste à "l'émiettement de son héritage" (p. 374). Zola avait déjà noté pour lui-même dans la reprise en 1883 de l'Ébauche de son roman: "Bien appuyer sur l'émiettement de la volonté [...], étudier dans un être l'émiettement que j'ai souvent étudié pour les choses [...], un vrai émiettement mangeant un être" (Hamon, 2005: 2021).

Zola semble vouloir confronter le lecteur avec un dilemme: comment doit-on se positionner face au processus destructif de l'émiettement et devant celui de la régularité stable des cycles naturels? Doit-on pencher, comme le fait Pauline, pour la régularité de ce dernier?

On le voit, la mer devient dans La joie de vivre, le personnage principal et omniprésent qui s'impose, aux yeux du lecteur, comme une présence constante due à ses mouvements réguliers dans le temps (la marée montante, la marée descendante, les grandes marées, les équinoxes) et dans l'espace (comme ligne ou barre à l'horizon). Incarnation des traits principaux associés aux milieux zoliens dans la plupart de ses romans,

\footnotetext{
${ }^{9}$ Qu'on pense, à ce propos, aux maladies ankylosantes trouvées dans quelques œuvres comme l'hydrarthrose dans Germinal qui empêche les mineurs de continuer leur travail, ou la paralysie qui cloue Phasie, dans la Bête humaine, à un fauteuil face au mouvement perpétuel des trains de la ville.
} 
à la fois mouvants et statiques, la mer rentre définitivement dans l'assemblage des milieux complexes qui déterminent largement le destin des êtres humains. Tel est le portrait dressé par Lazare, écrasé sous son poids:

Cette mer, avec son éternel balancement, son flot obstiné dont la houle battait la côte deux fois par jour, l'irritait comme une force stupide, étrangère à sa douleur, usant là les mêmes pierres depuis des siècles, sans avoir jamais pleuré sur une mort humaine. C'était trop grand, trop froid, et il se hâtait de rentrer, de s'enfermer, pour se sentir moins petit, moins écrasé entre l'infini de l'eau et l'infini du ciel (Zola, 2005: 291-292).

La disparité entre les dimensions de l'homme, terriblement petit et impuissant, et celles de la mer, infiniment vaste et écrasante, accentue la tragédie de l'homme sur terre ainsi que la fatalité du ciel et des éléments naturels, tel l'eau, qui pèsent éternellement sur son espèce. Beaucoup plus que le milieu socio-économique avec ses contingences qui caractérise la plupart des romans zoliens, le milieu naturel tel qu'il est peint dans La joie de vivre finit par projeter l'individu dans une dimension psychologique et philosophique sur la place de l'homme dans l'univers et lui ouvrir les horizons à ce que Zola avait appelé au début de son projet une "vaste névrose". 


\section{Bibliographie}

CHEVALIER, Gheerbrant (1982). Dictionnaire de symboles. Paris: Robert Laffont.

GONCOURT, Edmond et Jules de (1956). Journal. Tome III, 1879-1890. Paris: Fasquelle-Flammarion.

HAMON, Philippe (2005). Préface in La joie de vivre. Paris: Librairie Générale française.

RIPOLL, Roger (1981). Réalité et mythe chez Zola. Paris: Champion.

SERRES, Michel (1975). Feux et signaux de brume. Zola. Paris: Grasset et Fasquelle.

ZoLA, Émile (1895). Les romanciers naturalistes. Edmond et Jules de Goncourt. Paris: Bibliothèque Charpentier.

ZOLA, Émile (2005). La joie de vivre. Paris: Librairie Générale française. 